\title{
How to Integrate Predictions in Outcomes in Planning Clinical Care
}

\author{
Michael J. Germain \\ Baystate Medical Center and Tufts University, Springfield, Mass., USA
}

\author{
Key Words \\ Prognosis · Mortality · CKD · ESRD · Dialysis - Shared decision \\ making - Quality of life - Palliative care - Supportive care . \\ Elderly· Frailty
}

\begin{abstract}
Background: The CKD population is becoming increasingly elderly with multiple comorbidities. For this reason, accurate predictive information related to the progression into ESRD, mortality, and functional decline is critical to allow for optimal shared decision making (SDM). Summary: This review will assess the current literature on the methodologies for the estimation of prognosis and prognostic tools developed for CKD. A practical clinical approach is discussed that involves the estimation of prognosis and integration of prognosis into SDM. Key Message: There are validated, easy-touse prognostic tools that help clinicians engage in effective shared decision making with their CKD patients and family.
\end{abstract}

๑) 2015 S. Karger AG, Basel

\section{Introduction}

Nephrologists need to predict their patients' chances of future events to best manage their care with shared decision making (SDM) [1]. Prediction (prognosis) utilizes both the clinician's experience and the patient's knowledge with evidence from the literature [2]. Nephrologists and patients will benefit from predictions of; progression into renal deterioration (ultimately ESRD), mortality,
(C) 2015 S. Karger AG, Basel

0253-5068/15/0393-0065\$39.50/0 morbidities, illness trajectory, and quality of life. The communication of this estimate facilitates shared-decision making, often drives treatment decisions, and allows for patient-centered care. We know that our patients want to receive prognostic information even if it what they hear is not very encouraging [3].

Elderly multi-morbid patients, the highest incident dialysis population, experience high morbidity and mortality with the initiation of renal replacement therapy (RRT) $[4,5]$. These patients may not experience the benefits of dialysis that the younger healthier receive such as an acceptable quality of life and significant survival advantage. Conservative management $(\mathrm{CM})$, defined as chronic kidney disease (CKD) care without dialysis and with involvement of palliative care (PC) services, may be a better option for many of these patients [6-11]. Accurate shortterm (6-12 months) prognostic information such as the likelihood of progression to ESRD, mortality, and functional decline is critical to inform the clinician, patient, and family in the SDM process. Recently, many nephrologists have recognized that a patient-centered rather than a disease-centered approach is more appropriate to the care of these patients [12-16].

There are several prognostic tools that predict renal progression, short-, and long-term prognosis. Clinical parameters considered include, but are not limited to, functional status, nutritional status, comorbidities, and age. The Renal Physicians Association's (RPA) SDM guidelines endorse the communication of prognosis to all patients with acute kidney injury (AKI), CKD, and ESRD [1].
Michael J. Germain 


\section{Prognostic Tools}

Prognostic tools are risk prediction models that estimate the probability of having a disease (diagnostic prediction model DPM), or for developing a certain outcome (prognosis prediction model PPM) based on various patient variables [17]. DPMs and PPMs can be tools to help guide SDM.

Discrimination measures the model's accuracy defined by the area under the receiver-operating curve (AUC) in a logistic model, and the concordance, or cindex in a survival model [17]. Calibration measures the difference between observed vs. predicted frequency of the target event [17]. Validation is done by testing the model in a separate cohort [17].

External validity tests the model's generalizability to populations beyond the study subjects [18]. Generalizability is described by reproducibility or internal validity discussed above, and transportability, the accuracy of the model in a different, but related population [19]. There are five categories of transportability that aid clinicians in determining if a study is applicable to their patients: historical, geographic, methodological, spectrum, and follow-up [19]. A CPM or PPM can then be incorporated into a practical tool for the clinician; this can be in the form of a mobile app or integrated into decision support in an electronic medical record (EMR) [20]. Testing the benefits and risks of using the tool then allow clinicians to decide if they should incorporate it into their practice.

A prognostic tool can utilize a clinician's CPS (and/or patient's prediction) combined with a demographic, laboratory, functional status to form an integrated prognostic model (IPM) [21, 22]. Patients who are followed by nurses more than doctors are overly optimistic about survival. However the 'surprise question' (SQ) (would I be surprised if the patient dies in the next 6 [12] months), outperforms all other laboratory and demographic variables by 2-4 times [23]. Many medical guidelines recommend the use of the SQ [1]. The accuracy of CPS as death gets closer has been established [24]. The formulation of prognosis for a patient with renal disease involves a CPS (the SQ) integrated with laboratory data such as nutritional parameters, phosphorous, urine albumin to creatinine (UAC) ratio, hemoglobin, parathyroid hormone (PTH), estimated glomerular filtration rate (GFR), functional status, and patient subjective scores for parameters such as nutrition and comorbidity score. Sentinel events (hospitalization, MI, CVA, AKI, fractures, and changes of variables) over time can significantly improve the model's performance. A recent review notes that few CPMs or
PPMs met the standards of performance that would be required to allow for strong recommendations for their use [25]. Despite this fact, it is better to use an imperfect tool, recognizing it's weaknesses to help in SDM than to not have any guidance with prognosis.

\section{Pre-ESRD CKD. The Use of PPMs}

Prediction of time to progression of CKD can be very useful in SDM. This is especially true for elderly patients with comorbidities. Such patients are much more likely to die than to reach ESRD. Prognostic tools can help when deciding when and how to prepare a patient for RRT or conservative management.

A recent review of 11 models of CKD progression found 4 had been externally validated [25]. The model developed by Tangri et al. describe 8 variables (age, gender, GFR, calcium, phosphorous, bicarbonate, UAC, and serum albumin) and easily calculates the 2- and 5-year risk for progression to ESRD [26] (available at http:// www.qxmd.com/calculate-online/nephrology/kidneyfailure-risk-equation). The model has not yet been validated externally or piloted in clinical practice. The Chronic Renal Impairment in Birmingham (CRIB) was externally validated; the clinically useful calculator is available at http://www.ctsu.ox.ac.uk/cribcalculator/ [27].

Prediction of mortality in pre-dialysis patients has been evaluated in some studies but none of the studies focused on evaluating the 6-12 months mortality, and none of the studies was validated externally. The abovementioned CRIB study modeled progression to both ESRD and mortality [27]. It is possible that novel bio markers could improve prognostic models/tools but whether they add independent value to the standard variables is unknown. Asymmetric dimethylargine (ADMA), interleukin 6 (IL-6), high sensitivity C-reactive protein (hsCRP), cystatin c, transforming beta growth factor 1 (TGF $\beta-1$ ), troponin 1, and N-terminal pro naturetic peptide (pro-BNP) were evaluated in the Canadian Study of Prediction of Risk of Evolution to Dialysis, Death, Interim Cardiovascular (CV) Events Over Time (CanPREDICT) study [28].

\section{Incident ESRD Patients Mortality Prediction}

Since the morbidity and mortality of patients in the first 3 months after initiating dialysis is extraordinary high, especially in the elderly, accurate prognosis, could
Germain 
be particularly useful in discussions creating individualized patient-centered goals of care. The Dialysis Outcomes and Practice Patterns (DOPPS) study of 4,802 incident $\mathrm{HD}$ patients showed that age (RR per 10 years = $1.53,95 \%$ CI 1.10-2.13), in combination with pre-dialysis poor functional status $(\mathrm{RR}=2.28,95 \% \mathrm{CI}=1.01-5.01)$, presence of heart disease ( $R R=2.23,95 \%$ CI 1.16-4.29), and catheter use as initial vascular access $(\mathrm{RR}=2.45,95 \%$ $\mathrm{CI}=1.37-4.38$ ) can predict 90-day survival on dialysis for patients with diabetes (AUC $=0.77$ ) [29].

A study from the French Renal Epidemiology and Information Network (FREIN) developed a prognostic point-scoring system for 6-month mortality in incident HD patients $\geq 75$. The inability to transfer is a strong risk factor for early mortality (odds ratio $[\mathrm{OR}]=2.3,95 \%$ $\mathrm{CI}=1.9-2.8$ ). Recently, it has also been shown that the hospice eligibility criteria (HEC), based on functional status, has good discrimination for 6-month mortality in incident dialysis patients $\geq$ age 85 (c-statistic 0.65) [30].

In addition to physical vulnerability, older patients frequently initiate dialysis in the hospital setting with temporal intensive procedures and prolonged hospital stays, which results in very high costs [31]. A prediction model for one-year mortality of incident dialysis patients that combines the location of dialysis initiation (intensive care unit, outpatient unit, inpatient ward), hospitalization in the last year, and socioeconomic group, with comorbidities such as congestive heart failure and hypertension has shown good calibration and discrimination (c-statistic 0.75) [32]. Hospitalizations themselves represent an amplified risk of mortality, and recently it has been shown to increase by a factor of 11 in the month prior to death in hemodialysis patients [33].

Prognostic information should be integrated into clinical assessment, particularly in the elderly, where recognition and evaluation of geriatric syndromes such as frailty and falls in the context of comorbidities can be highly predictive Early identification of incident dialysis patients who have a high risk of 6-month mortality and frequent hospitalizations may allow effective SDM to allow for withdrawal from dialysis and/or initiation of hospice care.

\section{Prevalent ESRD Patients: Mortality Prediction}

The adjusted all-cause mortality rates for prevalent ESRD patients are 6.5-7.9 times higher than the general population. In a recent international study, decline in serum albumin, interdialytic weight gain (IDWG), systolic blood pressure, and increases in c reactive protein (CRP) were all noted more than one year prior to death. Low intra dialysis $\mathrm{BP}$ and weight loss predict death a few months before it happens [34].

Integration of a CPS into prognostication for ESRD patients can increase the accuracy of prediction [35]. In dialysis patients, the SQ was associated with a 3.5 higher OR for 1-year mortality. The Karnofsky Performance Status $(69.7 \pm 17.1$ vs. $81.6 \pm 15.8)$ and comorbidity score (CCI of $7.1 \pm 2.3$ vs. $5.8 \pm 2.1$ ) are independent predictors of death. Similar results were seen in a study from Hong Kong of peritoneal dialysis (PD) patients where an answer of 'no' to the SQ question was associated with a HR for death of 3.594 compared with the 'yes' group (95\% CI 1.411-9.151) [36]. The SQ, combined with the actuarial measurements of albumin, presence of peripheral vascular disease (PVD), dementia, and age, has been shown to be predictive of 6-month mortality in hemodialysis patients (AUC $=0.77,95 \% \mathrm{CI}=0.72-0.82$ ) [35]. These five predictors are combined in a validated prognostic tool (http://touchcalc.com/calculators/sq. or http://www. qxmd.com/calculate-online/nephrology).

In the case of the elderly especially, discussions of prognosis not only allow for informed decision-making, but also for advance care planning (ACP) for resuscitation and further interventions in the future. This is especially true in prevalent ESRD patients receiving RRT, for whom along with initial treatment-decision conversations, each sentinel event represents a time to revisit treatment-decision conversations and ACP [37]. As a patient's disease trajectory evolves, their prognosis as well as their wishes may change. A nephrologist can use prognostic tools to determine where in their disease trajectory their patient is. The typical disease trajectory of the elderly dialysis patient is a slow steady functional decline, punctuated by sentinel events such as hospitalizations In a cohort of elderly patients (age $>75$ ) with ischemic heart disease, the survival benefit of dialysis does not appear to result in increases survival compared to CM [9].

Protein malnutrition, low serum albumin, and poor appetite is associated with increased mortality in dialysis patients. A five-point scale appetite question to patients has been independently associated with mortality (RR of 4.74 (95\% CI 1.85-12.16)) and hospitalizations [38].

CKD patients have described a preference to have endof-life discussions with their nephrologists; yet nephrologists report being underprepared to have such conversations [39]. Particularly in the aging, highly comorbid and medically complex patient, preemptive discussion of pre- 
Table 1. How to communicate prognosis to our patients

1. Estimate prognosis of progression of CKD and survival (http://www.qxmd.com/calculate-online/nephrology).

2. Assess the patients' values and goals.

3. Assess your patients understanding of their disease progression and prognosis for survival.

4. Identify if your patient wants to hear his/her prognosis or to whom they would want to have that information communicated (proxy, surrogate).

5. Empathic communication of prognosis using non-medical terms, if speaking of survival use ranges instead of exact percentages or times (i.e., Hours to days, days to weeks, weeks to months). Use warning shots before delivery of bad news. ('I'm afraid the situation is more serious than I thought.')

Using SDM techniques and available RPA toolkit (https://itunes.apple.com/us/app/rpa-sdm-toolkit/id843971920?mt = 8), allow for an informed decision and treatment plan that is alignment with the patient's values.

6. Document ACP if the patient is ready to make these decisions. Patient support tools such as videos (http://www.acpdecisions. org/) can be helpful as well.

7. If a patient chooses $\mathrm{CM}$, elicit collaborate with palliative care specialists for an integrative approach to the patient's care.

Many additional resources are available:

The Coalition for Supportive Care for Kidney Patients website (http://www.kidneysupportivecare.org/Home.aspx).

The geriatric nephrology section of the ASN website (http://www.asn-online.org/education/distancelearning/curricula/geriatrics/). The RPA website (http://www.renalmd.org/practice-curriculum/) (http://education.renalmd.org/visitor_class_catalog/show/23567). Supportive Care for The Renal Patient Ed. Chambers J Brown E Germain MJ Oxford Press 2nd ed 2010.

dicted survival with and without RRT can guide the clinician to recommend CM in high-risk patients if this is consistent with an individual's preferences and goals Survival in those who start dialysis versus CM may modestly extend life, but often at the expense of the quality of life and those days of life are spent on dialysis, traveling to and from dialysis, and in hospital or rehabilitation units [7-11]. Many of our patients tell us that they were not prepared for the realities of dialysis and would not chose dialysis if they had the choice again. Nephrologists tell us that they are uncomfortable discussing prognosis and ACP with patients and family [39]. When there is enough uncertainty and with SDM consensus is lacking, a timelimited trial has been recommended $[1,43]$.

\section{Conclusion}

Accurate estimation of prognosis is the essential first step in SDM [1]. Studies have shown that our patients want to know their prognosis even if it is bad news. They feel it is important information to help guide their decisions on how 'aggressive' levels of care should be [40]. In order to accomplish this, we need to improve our prognostic tools [17]. Some of our colleagues point to the uncertainties and inaccuracy of using a population-based estimate of prog-

nosis in an individual patient as a reason not to have these discussions. Even so, currently available tools can assist in our SDM discussions with patients and family.

Once clinicians have estimated the patient's prognosis, they have to decide how best to convey this to the patient and family. Currently clinicians have very little guidance from evidence-based research on how to have these discussions. Yet there are some excellent resources that can help guide these discussions table $1[41,42]$.

Ongoing and future research will improve, validate, increase transportability, and generalize prognostic models to diverse populations. I believe we need to and will improve and validate our communication tools so that clinicians can lead effective SDM and provide patientcentered care.

References

Blood Purif 2015;39:65-69 DOI: $10.1159 / 000368946$
1 Renal Physicians Association: Shared Decision-Making in the Appropriate Initiation of and Withdrawal from Dialysis, ed 2. Rockville, Maryland, 2010.

$\checkmark 2$ Glare PA, Sinclair CT: Palliative medicine review: prognostication. J Palliat Med 2008;11: 84-103.

3 Davison SN: End-of-life care preferences and needs: perceptions of patients with chronic kidney disease. Clin J Am Soc Nephrol 2010; 5:195-204. 
4 US Renal Data System: USRDS 2012 Annual Data Report: Atlas of Chronic Kidney Disease and End-Stage Renal Disease in the United States. Bethesda, MD: National Institutes of Health National Institute of Diabetes and Digestive and Kidney Diseases, 2012.

5 Kurella M, Covinsky KE, Collins AJ, et al: Octogenarians and nonagenarians starting dialysis in the United States. Ann Intern Med 2007;146:177-183.

6 Jassal SV, Chiu E, Hadunewich M: Loss of independence in patients starting dialysis at 80 years of age or older. N Engl J Med 2009;361: 1612-1613.

7 Schell JO, Da Silva-Gane M, Germain MJ: Recent insights into life expectancy with and without dialysis. Curr Opin Nephrol Hypertens 2013;22:185-192.

-8 O'Connor NR, Kumar P: Conservative management of end-stage renal disease without dialysis: a systematic review. J Palliat Med 2012;15:228-235

-9 Murtagh FE, Marsh JE, Donohoe P, Ekbal NJ, Sheerin NS, Harris FE: Dialysis or not? A comparative survival study of patients over 75 years with chronic kidney disease stage 5 . Nephrol Dial Transplant 2007;22:1955-1962.

10 Carson RC, Juszczak M, Davenport A, Burns A: Is maximum conservative management an equivalent treatment option to dialysis for elderly patients with significant comorbid disease? Clin J Am Soc Nephrol 2009;4:16111619.

11 Da Silva-Gane M, Wellsted D, Greenshields $H$, Norton S, Chandna SM, Farrington K: Quality of life and survival in patients with advanced kidney failure managed conservatively or by dialysis. Clin J Am Soc Nephrol 2012;7:2002-2009.

12 Vandecasteele SJ, Kurella Tamura M: A patient-centered vision of care for ESRD: dialysis as a bridging treatment or as a final destination? J Am Soc Nephrol 2014;25:16471651.

13 Churchill DN, Jassal SV: Dialysis: destination or journey. J Am Soc Nephrol 2014;25:16091611.

14 Bowling CB, O’Hare AM: Managing older adults with CKD: individualized versus disease based approaches. Am J Kidney Dis 2012; 59:293-302.

15 Tamura MK, Tan JC, O’Hare AM: Optimizing renal replacement therapy in older adults: a framework for making individualized decisions. Kidney Int 2012;82:261-269.

16 Grubbs V, Moss AH, Cohen LM, Fisher MJ, Germain MJ, Jassal SV, Perl J, Weiner DE, Mehrotra R: A Palliative Approach to Dialysis Care: A Patient-Centered Transition to the End of Life. Clin J Am Soc Nephrol 2014 DOI: 10.2215/CJN.00650114.
17 Hendriksen JM, Geersing GJ, Moons KG, de Groot JA: Diagnostic and prognostic prediction models. J Thromb Haemost 2013;11(suppl 1):129-141.

18 Moons KG, Royston P, Vergouwe Y, Grobbee DE, Altman DG: Prognosis and prognostic research: what, why, and how? BMJ 2009; 338:B375.

19 Justice AC, Covinsky KE, Berlin JA: Assessing the generalizability of prognostic information. Ann Intern Med 1999;130:515-524.

20 Rigatto C, Sood MM, Tangri N: Risk prediction in chronic kidney disease: pitfalls and caveats. Curr Opin Nephrol Hypertens 2012;21: 612-618.

21 Cochrane Prognosis Methods Group: Scope of Our Work; Available from: http://prognosis methods.cochrane.org/scope-our-work (cited May 25, 2014).

22 Royston P, Moons KG, Altman DG, Vergouwe Y: Prognosis and prognostic research: developing a prognostic model. BMJ 2009; 338:B604.

23 Moss AH, Ganjoo J, Sharma S, Gansor J, Senft $S$, Weaner B, et al: Utility of the 'surprise' question to identify dialysis patients with high mortality. Clin J Am Soc Nephrol 2008;3: 1379-1384.

24 Chow E, Harth T, Hruby G, Finkelstein J, Wu J, Danjoux C: How accurate are physicians' clinical predictions of survival and the available prognostic tools in estimating survival times in terminally ill cancer patients? A systematic review. Clin Oncol (R Coll Radiol) 2001;13:209-218.

25 Tangri N, Kitsios GD, Inker LA, Griffith J, Naimark DM, et al: Risk prediction models for patients with chronic kidney disease: a systematic review. Ann Intern Med 2013;158: 596-603.

26 Tangri N, Stevens LA, Griffith J, Tighiouart $\mathrm{H}$, Djurdjev O, Naimark D, et al: A predictive model for progression of chronic kidney disease to kidney failure. JAMA 2011;305:15531559.

27 Landray MJ, Emberson JR, Blackwell L, Dasgupta T, Zakeri R, Morgan MD, et al: Prediction of ESRD and death among people with CKD: the Chronic Renal Impairment in Birmingham (CRIB) prospective cohort study. Am J Kidney Dis 2010;56:1082-1094.

28 Levin A, Rigatto C, Brendan B: Cohort profile: Canadian study of prediction of death, dialysis and interim cardiovascular events (CanPREDDICT). BMC Nephrol 2013;14:121.

29 Bradbury BD, Fissell RB, Albert JM, Anthony MS, Critchlow CW, Pisoni RL, et al: Predictors of early mortality among incident US hemodialysis patients in the Dialysis Outcomes and Practice Patterns Study (DOPPS). Clin J Am Soc Nephrol 2007;2:89-99.
30 Couchoud C, Labeeuw M, Moranne O, Allot $V$, Esnault V, Frimat L, et al: A clinical score to predict 6-month prognosis in elderly patients starting dialysis for end-stage renal disease. Nephrol Dial Transplant 2009;24:1553-1561.

31 Wong SP, Kreuter W, O’Hare AM: Healthcare intensity at initiation of chronic dialysis among older adults. J Am Soc Nephrol 2014; 25:143-149.

32 Cheung KL, Montez-Rath ME, Chertow GM, Winkelmayer WC, Periyakoil VS, Kurella Tamura M: Prognostic stratification in older adults commencing dialysis. J Gerontol A Biol Sci Med Sci 2014;69:1033-1039.

-33 Quinn RR, Laupacis A, Hux JE, Oliver MJ, Austin PC: Predicting the risk of 1-year mortality in incident dialysis patients: accounting for case-mix severity in studies using administrative data. Med Care 2011;49:257-266.

34 Usvyat LA, Kooman JP, van der Sande FM, Wang Y, Maddux FW, Levin NW, et al: Dynamics of hospitalizations in hemodialysis patients: results from a large US provider. Nephrol Dial Transplant 2014;29:442-448.

35 Cohen LM, Ruthazer R, Moss AH, Germain MJ: Predicting six-month mortality for patients who are on maintenance hemodialysis. Clin J Am Soc Nephrol 2010;5:72-79.

36 Pang WF, Kwan BC, Chow KM, Leung CB, Li PK, Szeto CC: Predicting 12-month mortality for peritoneal dialysis patients using the surprise' question. Perit Dial Int 2013;33:60-66.

37 Holley JL: Advance care planning in CKD/ ESRD: an evolving process. Clin J Am Soc Nephrol 2012;7:1033-1038.

38 Lukowsky LR, Kheifets L, Arah OA, Nissenson AR, Kalantar-Zadeh K: Nutritional predictors of early mortality in incident hemodialysis patients. Int Urol Nephrol 2014; 46:129-140. Available from: http://www. ncbi.nlm.nih.gov/pubmed/23703546.

39 Davison SN, Jhangri GS, Holley JL, Moss AH: Nephrologists' reported preparedness for end-of-life decision-making. Clin J Am Soc Nephrol 2006;1:1256-1262.

40 Davison SN: Facilitating advance care planning for patients with end-stage renal disease: the patient perspective. Clin J Am Soc Nephrol 2006;1:1023-1028

41 Schell JO, Green JA, Tulsky JA, Arnold RM: Communication skills training for dialysis decision-making and end-of-life care in nephrology. Clin J Am Soc Nephrol 2013;8:675-680.

42 Schell JO, Patel UD, Steinhauser KE, Ammarell N, Tulsky JA: Discussions of the kidney disease trajectory by elderly patients and nephrologists: a qualitative study. Am J Kidney Dis 2012;59:495-503.

43 Quill TE, Holloway R: Time-limited trials near the end of life. JAMA 2011;306:14831484.
How to Integrate Predictions in

Outcomes in Planning Clinical Care
Blood Purif 2015;39:65-69 